\title{
Occupation and chronic obstructive pulmonary disease
}

\section{To the Editor:}

In a recent editorial Dr. Sherwood Burge reviewed the evidence for the relationship between occupational exposures to dusts, gases and fumes and chronic obstructive lung disease [1]. We welcome Dr Burge's attention for this important but often under-appreciated subject. In the same issue of the journal our paper "Occupational exposures estimated by a population specific job exposure matrix and 25 year incidence rate of Chronic Nonspecific Lung Disease (CNSLD): the Zutphen Study" Post et al., [2] was published. Dr Burge commented the results of this study, and referred to our earlier work, also based on statistical analyses of the Zutphen Study, the Dutch contribution to the Seven Countries Study. He mentioned that "...It is disappointing that the results of the reanalysis are less clear than the original analysis, perhaps because of the lack of differentiation between the proportion of the workforce exposed to a particular agent and its intensity...". We would like to take the opportunity to comment on this statement.

The Zutphen Study was designed to study the relationship between diet and cardiovascular diseases. Also CNSLD epidemiology could be studied since information on respiratory symptoms, lung function and respiratory morbidity and mortality was gathered over the years. We started using these data in the late eighties to study relationships between occupation, occupational exposures and CNSLD [3, 4]. This early work demonstrated a clear relationship between occupational exposures to dusts, gases, and fumes and incidence of Chronic Non-Specific Lung Disease after 25 years of follow-up $(R R=1.4$ C.I. 1.07-1.85) [4]. This finding was in agreement with other studies, mostly cross-sectional. These studies have been performed in several countries e.g. Italy, Norway, France, Poland and the USA [5].

We reported earlier that workers exposed to dusts, gases and fumes tended to change job more often than those without an occupational exposure [4]. Those who changed jobs, changed to jobs which had a lower exposure or were physically less demanding. This would lead to the conclusion that risk ratio's might have been underestimated, because of this selective migration. However, it was unclear whether this was true and by what magnitude. We therefore undertook the study by Post et al. For this study we reconstructed the full occupational history from the beginning of this century until 1985 (end of follow-up) and developed a cohort specific Job Exposure Matrix.

We compared this matrix with the BMRC matrix and a well known US matrix for general population studies. These two matrices give also estimates of the intensity of exposure and showed an acceptable agreement with our own job exposure matrix based on probability of exposure [6]. This contradicts Dr. Burge's suggestion that there might be lack of differentiation between the proportion of the workforce exposed to a particular agent and its intensity. The most important aspect of the analysis by Post et al., however, was not the use of a cohort specific job exposure matrix but the fact that we applied an approach common in cancer epidemiology, but not yet applied in respiratory occupational epidemiology. This approach consisted of studying the relationship between time related exposure variables such as "time since first exposure" and "time since last exposure" with CNSLD incidence. Those who ever had an exposure to dust or a high exposure to dust showed risk ratios of respectively 1.3 and 1.9. Results of the more elaborate analysis with time related variables showed that those workers more recently exposed to dust had statistically significant higher risk ratio's than those exposed further back in time. This risk ratio was 7.5 (C.I. 4.1-13.8) for the group exposed 1-30 yrs ago, and 0.9 (C.I. 0.4-2.3) when exposure started $>60$ yrs ago. In addition, duration of exposure showed a strong negative trend with the risk of developing CNSLD as well. Both findings are indicative of a strong healthy worker effect. It will remain a matter of further debate which risk ratio estimates the contribution of occupational exposures to the development of CNSLD best. However, results do show that higher risk ratio's will be found when we apply statistical approaches in the analysis which remove the influence of the healthy worker effect to some extent. These observations show that the paper by Post et al. does contribute to the understanding of the relationship between occupational exposure and CNSLD and that it gives a better insight into this complex relationship than our earlier work offered. These results also justify further study and the use of more complex approaches in the data analysis of other general population studies. Our data might temper some of the optimism about the usefulness of general population studies in studying relationships between occupational exposures and respiratory morbidity. However, such conclusions should be drawn with care. General population studies should be regarded as sampling frames which allow unbiased sampling. When a full occupational history is taken at the end of the follow-up period it is possible to allow for the influence of the healthy worker effect in the statistical analysis. However, such approaches in the analysis have not yet been applied by most investigators but seems warranted if results of our study are well appreciated.

\section{Heederik}

Wageningen Agricultural University, Wageningen, The Netherlands. 


\section{References}

1. Burge PS. Occupation and chronic obstructive pulmonary disease (COPD). Eur Respir J 1994; 7: 1032-1034.

2. Post WK, Heederik D, Kromhout H, Kromhout D. Occupational exposures estimated by a population specific job exposure matrix and 25-year incidence rate of chronic nonspecific lung disease (CNSLD): the Zutphen Study. Eur Respir J 1994; 7: 1048-1055.

3. Heederik D, Pouwels H, Kromhout H, Kromhout D. Chronic nonspecific lung disease and occupational exposures estimated by means of a job exposure matrix: The Zutphen Study. Int J Epidemiol 1989; 18: 382-388.

4. Heederik D, Kromhout H, Burema J, Biersteker K,
Kromhout D. Occupational exposure and 25-year incidence rate of non-specific lung disease: the Zutphen study. Int J Epidemiol 1990; 19: 945-952.

5. Heederik D, Pal TM. The contribution of occupational exposures to the occurrence of chronic nonspecific lung disease. In: Hirsch A, Goldberg M, Martin J-P, Masse $\mathrm{R}$, eds. Prevention of Respiratory Diseases. Lung biology in health and disease, Vol 69. Marcel Dekker, New York, 1993.

6. Kromhout H, Heederik D, Daldrup LM, Kromhout D. Comparison of the performance of two general jobexposure matrices in a study of lung cancer morbidity in the Zutphen cohort. Am J Epid 1992; 136: 698711. 